\title{
Corrosion control and reduction in sludge storing concrete tanks in wastewater treatment plants using the metal - ceramic FGM plates element
}

\begin{abstract}
Concrete is one of the oldest and most common key materials in the construction and maintenance of wastewater transitional structures, which is considered by the designers and contractors. Corrosive materials in wastewater such as hydrogen sulfide, etc. cause corrosion at the concrete level, which over time leads to reducing their strength and therefore concrete structures mechanism. On the other hand, the increasing advancement of using composites in various industries created the idea of creating a new generation of composite materials known as FGMs for the first time in 1984 in Japan. FGMs are composites, in which the microscopic properties change with the non-uniform, smooth, and steady distribution of its original composition. Therefore, these materials are usually in the category of inhomogeneous materials that are assumed to be isotropic. This paper aims to investigate the application of the structural element of metal - ceramic FGM plates to design the plate elements of the floor, wall, or ceiling in cylindrical and rectangular concrete tanks of wastewater treatment plants sludge storage in order to extend the life of structures against corrosion caused by wastewater instead of concrete tanks. In addition, the previous structural results of the application of metal-ceramic FGM plate $s$ are the consumed elements used in the wall and floor slab on the support or on the substrate is elastic or Pasternak.
\end{abstract}

Volume 3 Issue 4 - 2017

\author{
Vahid Abdolvahab \\ Najafabad Islamic Azad University, Iran
}

\begin{abstract}
Correspondence: Vahid Abdolvahab, PhD Student of Civil Engineering, Najafabad Islamic Azad University, Najafabad,
\end{abstract} Isfahan, Iran, Email vahidabdolvahab@yahoo.com

Received: July 14, 2017 | Published: September 20, 2017

Keywords: sludge storing concrete tanks, concrete corrosion, FGMS, tank wall plate element, tank bottom plate element

\section{Introduction}

With the development of machine life and due to ignoring other people interest, more types of pollution put every day the environment in serious danger. Wastewater is one of the causes of environmental pollution. Therefore, they should be collected and removed out of the cities. They initially should be refined and then, returned to circulation in nature. Shape, substance, and wastewater ducts are considered by designers depending on the type and amount of waste. ${ }^{1}$ The purpose of wastewater and transforming them into sustainable materials is disinfection and killing germs. All these things are done in the nature in a relatively long-term and close to several days automatically. The purpose of the construction of wastewater treatment plant and developing on the one hand, and accelerating the mentioned works and shortening the duration of refining up to several hours on the other hand, prevent contamination of natural water sources and the environment. $^{2}$

\section{The reason for using concrete in structures in wastewater transmission and treatment structures}

Reinforced concrete pipes are the cheapest pipes in the sewerage network. They are easy to make and their application is economic and particularly suitable for diameters greater than $60 \mathrm{~cm}$. The static resistance of these pipes can be good depending on the calculation and design. The roughness coefficient of the inner wall of these pipes varies depending on their shape. ${ }^{1}$ Concrete has been considered as the most economical and the most important materials from designers to construct artificial wastewater treatment plants regarding the wastewater treatment plant structures (synthetic wastewater refining in mechanical, biological, and chemical methods) including tanks and wastewater timed storage tanks, wastewater transfer channels, pumping stations, etc. for artificial refining process of wastewater due to the relative strength of concrete structures in wastewater corrosion than other materials.

\section{Evaluation of corrosion causes of concrete structures in wastewater environments}

Nowadays, the optimized operation and maintenance of urban networks and water treatment plants aiming to increase the useful life of network services and treatment has become one of the challenges in the water and wastewater industry. Corrosion at most of old and operating concrete structures is in wastewater environments have led to traffic, social, and environmental problems. Repair of damaged concrete structures by traditional methods is very difficult. Therefore, new methods of preventing corrosion have long been running in developed countries. ${ }^{3}$

The types of Corrosion at concrete structures in the wastewater have been raised in the following: 
i. Corrosion of concrete structures caused by the reinforced concrete permeability,

ii. Corrosion of concrete structures caused by the cracks,

iii. Corrosion of concrete structures caused by gas generated in the sewer or chemicals,

iv. Corrosion of concrete structures caused by the concentration of metals and industrial wastewater,

v. Corrosion of concrete structures caused by vegetation.

\section{Definition of FGMS materials}

FGMs are the new generation of composites, in which the microscopic properties change with the non-uniform, smooth, and steady distribution of its original composition. These changes may be in one dimension or in multiple dimensions. The two mentioned phases in this substance are not distinct from each other. There are two pure metal and ceramic levels in heat-retaining plate $s$ that are a combination of ceramics and metal and produce a desired combination of these two materials between these two levels. In this case, the thermal and mechanical properties continuously change. The result of these gradual changes is a highly efficient production that meets all the requirements expected of such a structure. Thus, they are called substance with uneven thickness properties. ${ }^{4}$

The main use of FGMs in improving the mechanical and thermomechanical properties of components is as follows:

a. Since the transfer of such materials from a substance to another substance is gradual, the concentration stresses than those with discontinuous changes decreases.

b. Thermal stresses in the substance reach the minimum amount. In addition, controlling the thermal stresses in critical areas will be possible where there is the maximum thermal stress in that part.

c. Starting plastic surrender and failure to mechanical and thermal loading occur with a lag.

d. Resistance of interface bands in heterogeneous solids, such as metals and ceramics increases with continuous changes in the composition or making the mechanical properties directional.

As mentioned, FGMs materials have different metallurgical structure. Therefore, they are considered as composite materials with microscopic heterogeneous properties, but continuous changes in the microstructure of FGMs materials makes them distinct from conventional composite materials. FGMs materials are made with conventional composite materials. Changes in FGMs substance is gradually and gradually is reduced from a substance and added to another substance. The most important advantage of this substance is that all the required properties such as flexibility and resistance to heat and corrosion exist in them without sacrificing one of them for another. Due to the required applications and functions, these materials are often made of two homogeneous substances usually a metal and a ceramic (Figure 1). Metal has properties such as ductility, high strength, stiffness, strength, and machining capabilities. On the other hand, ceramics have properties such as high strength to corrosion at wastewater substance.

\section{Ceramics}

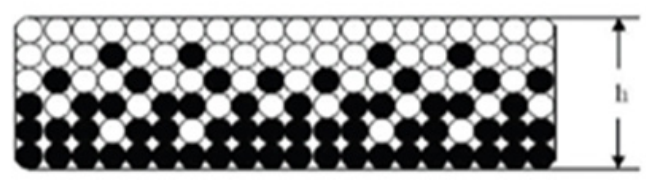

Metal

Figure I Schematic of FGM plates. $^{5}$

For the first time, Bewer \& Duwez et al., 5 raised the idea of combining two different phases by changing the arrangement of each of the phases in the layer to improve the mechanical properties of the composites. Their idea was generally related to eliminating the weaknesses of composite materials in many of their application. However, until 1980 no research has been done on the design, construction, evaluation, and analysis of substance with the gradual changing structure. In the mid-1980s, the scientific name of FGMs was first given to this substance in Japan and a new arena was opened for the extensive research on this type of substance. Their goal was to produce a substance that can withstand the temperature of $2000 \mathrm{Kas}$ well as the thermal gradient $1000 \mathrm{~K}$ in a thickness of less than $10 \mathrm{~mm}$. Since these materials are heterogeneous, conducting comprehensive studies in the fields of design, modeling, making, and evaluation was necessary and inevitable. ${ }^{7}$ At that time, a state-owned group in Japan predicted that the entry of Japan into the modern space research and development of these types of research depend on new materials. The three of scientists named Nino, Koizumi, and Hirray, started their research on the spaceship project, ${ }^{6}$ their studies showed that the applied structural components in the body of the spacecraft are affected by the extreme thermal loads. Therefore, it is necessary to consider two very important cases in combining and making the body infrastructures:

a. The structural components should be produced by available materials to achieve the maximum of the desired goals.

b. The stress concentration should be prevented by the proper connection of different materials.

c. During 1980 to 1990 out of Japan in several countries including Germany, the United States, China, and Russia, FGMs were the new subject of studies that are continuing. For example, in Germany, a six-year research program was arranged in Germany since 1995 and with the participation of a large number of laboratories.

\section{Applications of FGMs}

The extensive application of FGM materials is due to gradual changes of their properties because the mismatch problem in mechanical properties does not exist at the junction layers compared with the layered composite substance. This mismatch causes an increased willingness of separation in the layers under the extreme thermal loading. The residual stresses will be unavoidable due to the different thermal expansion coefficients of the layers. FGMs are widely used in the aerospace industry as well as in structures that are subjected to extreme changes in temperature and thermal shocks, such as thermoelectric devices used in energy conversion industry. Although 
this substance was originally designed for use in spacecraft structures and nuclear reactors, but in recent years found many applications including the connection of metal to ceramic, transplanting members to human, explosion engine components, magnetic means, cutting tools, fire extinguishers, polymer composites with high strength coating of combustion chamber of Rocket propellant, rocket launcher pocket primer, and making piezoelectric and ferroelectric materials. ${ }^{7}$

\section{Modelling of properties}

These materials are inhomogeneous. Therefore, their physical properties such as the modulus of elasticity and thermal conductivity vary. However, the structure of FGMs is such that the isotropic assumption is valid in most cases.

Generally, there are two different approaches to model changes in properties of these materials:

a. Assuming a function for the volume fraction changes of the substance

b. The micromechanical approach to the study of a heterogeneous environment

Various correlations have been so far proposed for modelling FGMs considering their type of structure. The dominant distribution in various studies for the made plate $\mathrm{s}$ by these materials, FGMs whose properties are only in terms of uneven plate thickness is defined as follows.

$$
\begin{gathered}
P(Z)=\left(P_{t}-P_{b}\right) V+P_{b} \\
V=\left(\frac{2}{h}+\frac{1}{2}\right)^{n} ;-\left(\frac{n}{2}\right) \leq Z \leq\left(\frac{h}{2}\right)
\end{gathered}
$$

In which, $P(Z)$ is a general feature such as modulus of elasticity or density. $\mathrm{Pt}$ and $\mathrm{Pb}$, respectively, are the profile of the upper and lower sides of the plate, and $\mathrm{h}$ is the total thickness of the plate. $\mathrm{V}$ is the volume fraction of the legal side substance of the plate. As shown in Equation (1B), it is assumed in the form of a power distribution. $\mathrm{N}$ is a parameter that shows the substance distribution in the thickness. For example, the changes are linear if the number is equal to one and it will be a homogeneous substance with the features of the upper side. The lower side substance increases by increasing the $\mathrm{N}^{2}$

\section{The sludge storage tanks using the FGM plates element}

The purpose of this section is to evaluate the conditions of using FGM plates with high corrosion resistance in wastewater environments as the wall and floor plate elements of concrete tanks. The intended FGM plates for using in the wall and floor elements of concrete tanks is a metal - ceramic composite plate with a gradual change in the thickness. The ceramic part is made of alumina and the metal part is made of aluminium. First, alumina corrosion is investigated in wastewater environments and aluminium corrosion is investigated in contact with air and water.

Valuation of alumina (ceramic parts) used in FGM plate $s$ to the internal wall of sludge storage tanks

Aluminium oxide or alumina $\left(\mathrm{Al}_{2} \mathrm{O}_{3}\right)$ is one of the important ceramic substance with a wide range of applications in various fields. Among the various and outstanding properties of alumina, the mechanical properties such as high compressive strength and high hardness as well as refractoriness can be mentioned. ${ }^{3}$

The maximum use of ceramic materials in wastewater environments is as follows:

The application of clay pipes to transfer wastewater: The excellent resistance of ceramic pipes to waste water corrosive acids and materials, low permeability of ceramic pipes to water, smooth surface with low roughness coefficient even lower than plastic pipes, good static resistance, cheap, and availability of raw materials i.e. clay can be one of the advantages of these pipes. ${ }^{4}$

Ceramic application to prevent corrosion at wastewater concrete structures: One of the methods for preventing concrete corrosion at European wastewater environments is the protection of its surface using ceramic glazes. The pre-built components glass clay tiles made by the European company with different dimension and size based on the coverage are used along with mortar from an epoxy resin layer by creating an exceptional bond and mechanical resistance with chemical resistance in the control of the designed wastewater. Corrosion problems have been resolved in the consumed mortar. Tile components (Figure 2) are appropriate for pipes with various cross sections such as circular, oval, and square. ${ }^{5}$ Ceramic materials are still the first option of concrete restoration in wastewater treatment plants especially for channel and sewer lines, but the main discussion is on how to install them. The mentioned ceramics can be applied to make prefabricated pipelines or to prevent corrosion at old pipes. The advantages of glazed ceramics can be named as follows:

a. Resistant to chemicals,

b. Resistant to water vapour and impermeability,

c. Hardening without cracks,

d. Resistant to freezing and weathering,

e. Production of connection levels always with low porosity,

f. Resistant to high corrosion. ${ }^{6,7}$

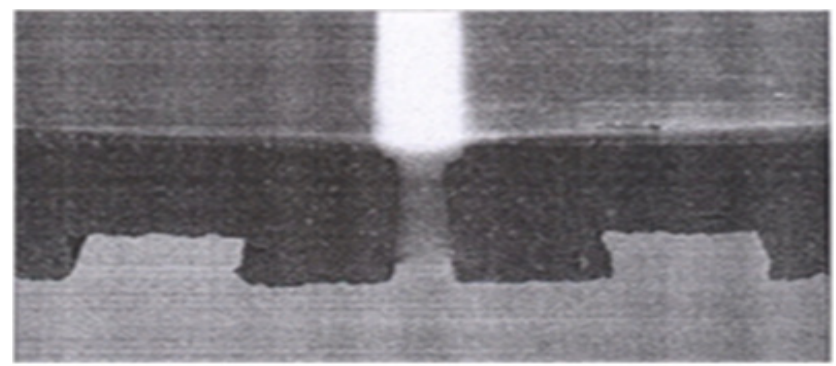

Figure 2 Consumer glazed tiles with lining materials. ${ }^{13}$

Applications of ceramic membranes in the oil waste water treatment: In recent years, the ceramic membranes are used in oil wastewater treatment processes due to many advantages such as high chemical heat resistance, long life, and washing facilities with various chemical materials. ${ }^{8}$ The mentioned cases indicate that the maximum use of ceramics in wastewater and corrosive chemical environments.

If the permeability of alumina in FGM plates causes water penetration in the ceramic layers; this problem can be met by Glazing the ceramic surface of FGM plates, which can control the permeability of the wastewater substance. 


\section{Evaluation of the consumed aluminium (metal) in FGM plates to the outer surface of the waste water storage tanks}

Aluminium is a soft and lightweight metal, but strong. It has a matt silver - gray appearance with a thin layer of oxidation, which is formed by the collision of air at the surface. It is malleable, flexible, and bends easily. It is also very durable and anticorrosive. In addition, this nonmagnetic element is the second malleable and the sixth ductile metal with no spark. Aluminium is the most applied metal after iron, which is important almost in all industry sectors whether in terms of quality or value. Pure aluminium is soft and weak, but it can create alloys with small amounts of copper, magnesium, manganese, silicon, and other elements. These alloys have a variety of useful properties. These important alloys make aircrafts and rockets. ${ }^{9}$

Aluminium corrosion at contact with air: Another property of aluminium alloy is resistance to corrosion, when the pure aluminium is exposed to air, it is immediately covered with an adhesive layer of aluminium oxide. This covering layer prevents the corrosion. If the layer is peeled off by wearing, it is immediately formed again. The thickness of this layer is normally about 0.01 micrometers. The mentioned layer is so sturdy that is an effective barrier against most corrosive materials. The Aluminium oxide layer exposed to the air has a good protection feature, but this oxide layer can be made by electrolysis. This is Anodization and the formed oxide is anodic oxide coating. Anodization creates a compressed layer near the metal surface and another layer with fine pores on it. ${ }^{10-13}$

Corrosion of aluminium in contact with water: A kind of aluminium corrosion occurs in the presence of water, which leads to a gap and forming aluminium oxide and bleaching the surface in the form of water spots. Dispelling water spots is difficult and probably impossible. Aluminium materials immersed in water can be retained by cathodic protection against the formation of cavities. To do this, the electrode potential comes down below the potential to form the desired holes in the body. However, the hydrogen gas can be formed at the cathode, which is resulted in increasing $\mathrm{PH}$. When $\mathrm{PH}$ goes very high, aluminium is likely to come under attack. Thus, the extra protection should be avoided. ${ }^{11-13}$

\section{The structural research survey of FGM plates for the consumed plate elements in wastewater storage tanks}

In recent years, a variety of structural types of research have been done due to the importance of the subject in the field of FGMs tank characteristics and behaviour as shown in (Figure 3).

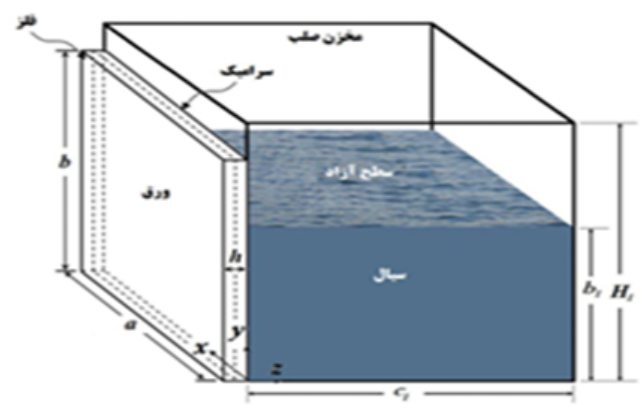

Figure 3 Application of FGM plates in cubic tank walls in wastewater treatment plants. ${ }^{18}$

\section{Evaluation of FGM plates elements to cubic and cylindrical tanks walls}

This section reviews the previous studies on the FGM plate element in tank walls against hydrostatic forces of the fluid and the force caused by disturbance of the fluid perpendicular to the middle cubic and cylindrical tank plate (Figure 4). Bakhasheshi \& Khorshidi et al., ${ }^{14}$ examined the free vibration of the rectangular plate made of functionally graded materials in cross contact with the limited turbulence fluid and they have applied simply animated and nonanimated support assuming the third order theory from harmonic sine functions for the rectangular tank wall.

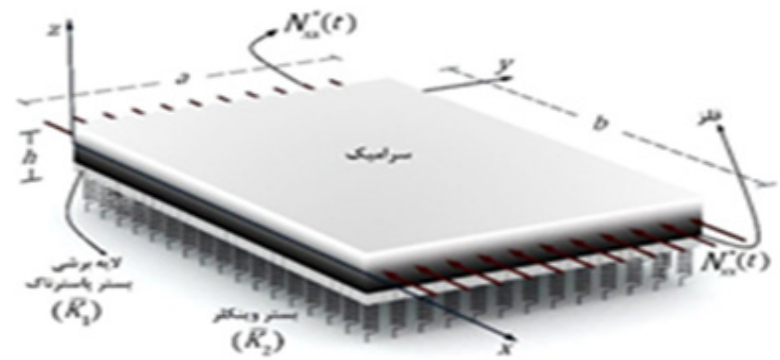

Figure 4 Bottom FGM plate on elastic Pasternak subjected to mechanical loads. ${ }^{28}$

By examining the numerical results, it was determined that:

a. Frequency values in contact with the air are more than a turbulent fluid.

b. The amount of vibration frequency will be reduced by increasing the plate side.

c. The frequency increases by increasing the plate thickness.

The vibration frequency will be less by increasing the depth of the fluid.

a. The plate vibration frequency increases by increasing the width of the tanks.

b. The basic frequency of vertical vibration system is coincided on the animated and non-animated simple support. It has also been observed that the vibration frequency for the fixed mode is before the animated and non-animated simple support.

c. The natural frequency of plates increases by increasing the volume fraction coefficient of the materials due to the increasing plate tightness. ${ }^{15}$

Tahani \&Talebian., ${ }^{16}$ analyzed the FGM conical tanks under mechanical loads symmetrical with two cantilevers support through the two-dimensional finite element method. According to the results, it was observed that the stress distribution in a cylindrical container made of pure metal or ceramic is only correlated with the tank geometry and load while the distribution of temperature stress in cylindrical tank made of pure metal or ceramic is only correlated with the tank geometry and load, materials, properties changes method. This dependence is to control the stress and temperature distribution. In addition, the use of FGMs in the construction of cylindrical tanks reduces the environmental stresses on the inner surface. The effect of this condition is very noticeable in heat loading than the mechanical loading. Tohidi et al., ${ }^{17}$ optimized the stress in the spherical tanks made of FGMs plates under heat and pressure loads. In this paper, 
an analytical solution was used to calculate the radial stresses of spherical tanks made of thick FGM plates. Finally, a general model was obtained to get the radius and circumference stresses in thickwalled spherical tanks with FGM materials.

Safari et al., ${ }^{18}$ evaluated the analytical method to study the dynamic response of a thick-walled cylindrical under mechanical shock so the Laplace domain equation was obtained using the series of cylindrical targeted and dynamic response in the time domain by using theoretical analysis of the residuals. Isvand Zibaei et al., ${ }^{19}$ investigated the effect of fixed-fixed and fixed-free boundary conditions for FGM cylindrical shell vibrations consisting of stainless steel and nickel with reinforced ring based on the third order theory of shear deformation. They concluded that the reinforced ring affects the frequencies. This effect will have the highest value compared to the ring and the boundary conditions of the cylindrical shell. When the reinforced ring moves from the shell centre to the bottom of the shell, the natural frequencies are reduced. Therefore, the natural frequency curve will be symmetrical around the centre of the shell. This symmetry is at both ends of the shell around the symmetrical shell centre.

\section{Evaluation of FGM plates elements to cubic and cylindrical tanks bottom on the edge supports}

This section reviews the previous studies on the FGM plates in the bottom of the tank (the ceramic top plate ) against the static forces of due to a uniform fluid pressure on the bottom (perpendicular to the middle-plate ) with edge supports for cubic and cylindrical tanks. For this purpose, the conducted studied on the rectangular and circular plates loaded under different conditions should be investigated. Abdolvahab.,$^{20}$ studied the Galerkin method to investigate the static behaviour of FGM plates used in ceiling and floor cubic tanks subjected to uniform uniaxial compressive loading under different boundary conditions. Heidari., ${ }^{21}$ examined the critical load of a circular FGM plate with clamped edges under various mechanical load on three theories of 1. Classic 2: The first order shear deformation, and Higher-order shear deformation. It was concluded that the critical load depends on the parameters of volume fraction, thickness, and radius so that increasing the plate's ratio of thickness to radius increases the mechanical strength. Generally, the high order theories responses are more accurate than the first-order and the classic theory.

Shareiyat et al., ${ }^{22}$ evaluated the non-linear analysis of the hollow circular plate deflection of functionally graded materials with variable properties in both the transverse and radial strain of the classical theory and Fan Carmen nonlinear shift -strain relationship. It was concluded that generally when the changes of properties are considered in both the radial and thickness directions, the maximum deflection is reduced by increasing the volume fraction modulus in the thickness and radial direction and its location moves towards the interior radius. Jafari \& Jandaqian., ${ }^{23}$ examined the forced vibrations of a circular FGM plate based on the classical theory and simple and clamped boundary conditions on the edge of the plate using the Bessel equation. They concluded that the volume fraction highly affects the deflection and frequency of the plate by increasing the volume fraction module of the plate, deflection, and natural frequency. Shoshtari et al., ${ }^{24}$ discussed the vibration of graded rectangular plates under the local broad mass and simple support using Hamilton's based on the third order shear deformation theory. They concluded that non-dimensioned frequency of the plate reduces by increasing the ratio of length to thickness and ratio of the mass and the frequency increases by increasing the ratio of length to width and the effect of the mass. However, the frequency decreases whatever the mass gets closer to the middle of the plate and Sigmund graded plate indicates more rigidity that increases the frequencies.

\section{Review of FGM plate elements for cylindrical and cubic tanks on the substrate}

This section reviews the previous studies on the FGM plate element in the bottom of the tank (ceramic side top of the plate - (Figure 4) against static forces caused by the uniform pressure of the fluid on the bottom located on different substrates such as elastic, Pasternak, plastic, or middle supports under FGM plates for cubic and cylindrical tanks. It is enough to investigate the conducted static analysis on the rectangular and circular plates with support conditions on different substrates. Rezaei \& Jahangiri., ${ }^{25}$ evaluated the dynamic and static instability as well as nonlinear vibrations of FG plates with the elastic substrate under stimulated parametric of a force exposed to harmonic forces inside a plate based on the classical theory and using the theory of Wen-Carmen and the Hamilton large deformations. It was found that the natural frequency increases by increasing the parameters of the elastic substrate and dynamic instability occurred at higher excitation frequencies. The plate is stimulated by the parametric excitation frequency in a certain resonance range so that the system will have two unstable and stable nontrivial periodic solution by provoking the setting parameter in a part of this provoking range.

In another part of the resonance range, the system will have a stable periodic nontrivial solution. Moreover:

i. FGM plate buckling occurs in the form of higher modes by increasing the substrate parameters and the corresponding buckling occurs with the absence of the elastic substrate in larger quantities than the critical force.

ii. By the parametric provoking the plate in the presence of the elastic substrate absence of damping effect; increasing the elastic substrate parameters resulted in approaching the subcritical and supercritical bifurcation towards each other, which leads to a reduction in the resonance range.

Bazkiaei \& Tarzjani., ${ }^{26}$ examined the FGM plate free vibration on the Winkler elastic substrate using differential quadrature element method. They found that elastic substrate with FGM plate makes changes in the modal parameters such as natural frequency. Rahimi et al., ${ }^{27}$ extracted the overall equation of the cylindrical thick-walled ax symmetric made of FG heterogeneous materials using the theory of plane elasticity. It was observed that the environmental stress reduces in one half and increases in another half for every positive or negative heterogeneity factor. The radial stress increases and the radial displacement decreases for each positive heterogeneity factor. The radial stress decreases and the radial displacement increases for each negative heterogeneity factor. Further changes can be observed in the larger absolute value of the heterogeneity factor. Thus, about $20 \%$ change in the behaviour of homogeneous material is seen for $+1,-1$, which can be used depending on the need for displacement or tension in the heterogeneous cylinder.

\section{Conclusion}

a. The use of ceramic is recommended in these environments considering the high ceramic resistance in acidic and sanitation environments. Therefore, using ceramic-metal FGM plate $\mathrm{s}$ is very convenient for the plate elements of the bottom, wall, and 
concrete tank bottom for wastewater storage so that the plate side is in contact with the wastewater.

b. According to the previous paragraph, if the ceramic is in contact with wastewater, the aluminum layer of the consumed FGM plate on the wall and bottom of the tank will be in contact with the concrete-wall, air, water, or soil. According to the presentations, aluminum is corrosion resistant in the mentioned environment complying with the measures.

c. Given that the FGM plate frequency values when contacting the plate with air are more than the contact with the turbulent fluid, thus this plate can be used reliably in contact with the turbulence fluid.

d. Given that the plate vibration frequency increases in contact with the turbulent fluid by increasing the FGM plate thickness and tank width, it can be concluded that it is better to use cylindrical concrete tanks wall instead of using these plate $s$ in the large concrete cube tank walls.

e. Considering that the plate deflection under loads perpendicular to the bottom of the tank in metal-ceramic FGM plates decreases by decreasing the modular plate volume fraction. It is suggested to use FGM plates with lower volume modular fraction in the bottom of the tank.

f. Given that upper side volume, modular fraction indicates the material distribution in thickness and the zero value shows a homogeneous material with an upper side profile (ceramic) and the volume modular fraction of a material with linear changes and the ceramic-metal volume modular fraction increases. Thus, it is recommended to use FGM plates with volume modular fraction range of zero to one in the tank walls and bottom to increases the thickness of the ceramic for corrosion resistance in wastewater environments.

g. Considering the fact that the critical buckling load of the plates increases with decreasing the volume modular fraction, it is recommended to use FGM plates with lower volume modular fraction in tank walls.

h. The use of elastic and Pasternak substrates increases the buckling load and decreases deflection in the FGM plate elements in the bottom of the tank.

i. The use of FGMs in the construction of cylindrical tanks reduces the environmental stresses on the inner surface. The effect of this condition is very noticeable in the heat loading than the mechanical loading.

\section{Acknowledgments}

None.

\section{Conflicts of interest}

The author declares that there are no conflicts of interest.

\section{Funding}

None.

\section{References}

1. Monzavi, Mohammad Taghi. Urban wastewater-wastewater collection. Iran: Tehran University Publications. 2010.
2. Monzavi, Mohammad Taghi. Urban wastewater-wastewater collection. . Iran: Tehran University Publications.2010.

3. Qiominia, Mojdeh. The introduction of modern methods of rehabilitation of damaged wastewater networks and their comparison with the performance index. Kerman, Iran: National Conference on Water and Wastewater Engineering. 2012.

4. Reddy JN. Mechanics of laminated composite Plates and shells: theory and analysis. Iran:CRC Press. 2004.

5. Mozafari H, Ayob A. Effect of Thickness Variation on the Mechanical Buckling Load in Plates Made of Functionally Graded Materials. Proceeding of Technology. 2012;1:496-504.

6. Bever M, P Duwez. Gradients in composite materials. Materials Science and Engineering. 1972;10:1-8.

7. Miyomoto Y. Functionally Graded Materials: Design, Processing, and Applications. Netherlands: Kluwer Academic Publishers. 1999.

8. Hirai T. Functional gradient materials. Processing of Ceramics Part 2. 1996;17B.

9. Wajih al-Sadat F, Azhari M. Evaluation of heat buckling in the thickness of the relatively thick plates with dissimilar properties. Semnan University, Iran: National Engineering Congress. 2011.

10. Farhoudi M. Comparison of technical and economic types of pipes used in sewage collection networks and the preferred option. Tehran, Iran: The third national conference on water and sanitation (with a view to reforming consumption patterns). 2009.

11. Abdul Wahab Vahid. Evaluation of corrosion of concrete structures and methods of prevention and treatment of wastewater in the environment. Journal of cement, concrete and Bnaryal. 2015;17:16-24.

12. Abbasi M. The application of ceramic membranes in oily waste water treatment and oil. Iran: The first conference of the refining technologies in the environment. 2011.

13. Einar Mattsson. the fundamentals of corrosion technology.

14. Alizadeh Tusi M. Corrosion of metals and preventation.

15. Bakhsheshi A, Khorshidi K. Plate in contact with the fluid. Journal of Mechanical Engineering lecturer. 2014;14(8):165-173.

16. Tahmani M, Talebian T. Analysis of conical tanks made of functionally graded materials (FGMs) under mechanical and thermal loads. Journal of Mechanical Engineering Technology, forty-first year. 2009;1:49-59.

17. Sadeghi Tohidi, Farzad Karen Ebarinia, Hassan Naei, et al. Analysis of stresses in cylindrical thick-walled FGM under loads of pressure and heat. The second International Conference and the Eighth National Conference on Manufacturing Engineering. 2007.

18. Safavi Ali, Masoud Tahmani, Seyed Mahmoud, et al. Thick-walled cylindrical dynamic stress analysis of FGM under mechanical shock to the analytical method. XVIII Annual Conference of Mechanical Engineering. 2010.

19. Isvand Zibaei M, Jahani A. Effects of boundary conditions and clampedclamped and clamped-free for free vibration FGM cylindrical shell with reinforced rims, based on third-order shear deformation theory. Journal of aerospace mechanics (dynamics, vibration control). 2010;6(3):25-38.

20. Abdolvahab V. Stability Analysis of Cube Tanks' Ceiling and Floor Plates Composed of FGMs when Subjected to Uniform Compression Perpendicular to the Mid-Plane. MOJ Civil Eng. 2017;3(1):00059.

21. Hamid Reza Heydari. The theory of higher-order shear deformation theory of first-order shear deformation theory and classical circular plate FGM with the support clamped under the uniform mechanical. India: The second National Conference on Development and Engineering Sciences Branch. 2015. 
22. Shariat M, Dashtabadi Gh, Jafari A. Nonlinear Analysis prone plate circle with properties varied in both the transverse and radial. Journal of Mechanical Aerospace. 2013;9(4):35-49.

23. Jafari A, Jandaqian A. Evaluation of FGM circular vibrating plate using Bessel functions. Iran:The Nineteenth Annual Conference of Mechanical Engineering Iran. 2011.

24. Shoshtari A, Motahari Z, Kari M. Vibration of graded plates under the broad mass of the second-order shear theory. Journal of Applied and Computational Mechanics. 2015.

25. Rezaei M, Jahangiri R. dynamic and static instability as well as nonlinear vibrations of FG plates with the elastic substrate under stimulated parametric of a force. Journal of Mechanical Engineering lecturer. 2014;14(13):172-182.
26. Bazkiaei A, Tarzjani H. Evaluation of FGM plate free vibration on the Winkler elastic substrate using differential quadrature element method. The second National Conference on Mechanical systems and industrial inventors Ahvaz, Islamic Azad University, Iran. 2013.

27. Rahimi GH, Qanad M, Esmaeilzadeh Kham S. General solution of cylindrical thick-walled axisymmetric made of heterogeneous materials FG using the elasticity plane. Journal of Engineering lecturer-in Mechanical Engineering. 2010;10(3):31-43. 